\title{
THE COST OF OPPORTUNITY FOR GAPLESS IMAGING
}

\author{
Marwan Younis $^{\star}$, Felipe Queiroz de Almeida ${ }^{\star}$, Sigurd Huber ${ }^{\star}$, \\ Mariantonietta Zonno ${ }^{\star}$, Marc Rodriguez-Cassola $^{\star}$, Scott Hensley ${ }^{\ddagger}$, and Gerhard Krieger ${ }^{\star}$ \\ ${ }^{\star}$ German Aerospace Center (DLR), Oberpfaffenhofen, Germany \\ $\ddagger$ Jet Propulsion Laboratory, California Institute of Technology, Pasadena, USA.
}

\begin{abstract}
Utilizing digital multi-channel technology, spaceborne synthetic aperture radar instruments are capable of imaging swath widths of hundreds of kilometers at fine azimuth resolution. The main benefit follows through the extension of the trade space and the use of new digital beam-forming techniques facilitated through the multi-channel instrument architecture. This is truly a quantum leap as the performance of these systems will be orders of magnitude better than current in-orbit and state-of-the art systems. One of the basic restrictions applicable to spaceborne platforms hosting both the transmitter and receiver is the "blinding" of the receiver during the transmit time instances, which manifests itself through imaging gaps. One of the main challenges the instrument designers are faced with, is to circumvent these gaps, requiring the use of dedicated instrument operation modes. An alternative approach is multi-beam imaging, i.e. to allow the gaps in the single SAR acquisition, while using an appropriate mission design for filling the blind gaps. This paper explores the trade space options for high-resolution wideswath SAR imaging. The comparison of multi-beam and gapless imaging from an instrument design and performance point of view is elaborated.
\end{abstract}

\section{INTRODUCTION}

The basic idea behind digital beam-forming SAR is to sample the received signal on a sub-aperture level and record the data of the individual digital channels. In comparison, a "conventional" SAR instrument samples the weighted sum of the sub-apertures' signals, resulting in a single output data stream (for single polarization operation). The benefit of using multiple digital channels is to extend the trade-space of SAR, which by using appropriate operation modes or techniques, yields a significantly improved performance [1].

A restriction that remains, is that a SAR instrument cannot receive while it is transmitting, as the high transmit power (in to order of several kilowatts) would damage the receive hardware designed to detect extremely weak echo signals ${ }^{1}$. Thus, the signal echos arriving during the time instances in which the instrument is transmitting will not be recorded resulting in blind ranges along the swath. The width of these blind ranges is proportional to the transmit pulse duration and varies with the incidence angle. For a typical multi-channel SAR operating in stripmap mode to image a swath of $400 \mathrm{~km}$ the width of the gaps is in the order of a few to tens of kilometers, while, in single pol, the SAR image would contain 3 to 5 such gaps at distinct ranges.

Two main mitigation strategies are known: either use appropriate techniques to prevent gaps from appearing in the processed SAR image; or allow gaps in the single acquisition and use successive acquisitions to "fill" the gaps. Thus following an instrument/mode or a mission design based solution approach, respectively. It is interesting to note that favorable solutions, e.g. [3], seem to arise from different points of view of the same phenomenon. Considering either an ultra wide swath containing gaps or multiple non-contiguous swathes.

It is the aim of this paper to raise the awareness for the consequences of each solution strategy. In this sense, the "cost of opportunity" relates to the price paid for choosing one solution versus the other.

\section{BASIC MULTI-CHANNEL TECHNIQUES}

The following two techniques can be understood as the basic multi-channel building blocks. On one hand these can be combined with conventional operation modes such as stripmap, ScanSAR or TOPS, and on the other hand be extended to new more advanced techniques.

\footnotetext{
${ }^{1}$ Applies to systems which host both transmitter and receiver on the same platform. Future SAR constellations consisting of a few transmit-only and several receive-only satellites [2] allow even continuous transmission without blinding the receivers.
} 


\subsection{SCan-On-REceive (SCORE)}

Multiple channels in elevation allow generating a receive beam which follows the SAR signal echo while it traverses the ground. This technique, known as SCORE [4], allows an effective increase in the receive antenna elevation dimension in order to generate a narrow and high gain beam; this yields a higher signal-to-noise ratio and allows for the better suppression of range ambiguities. The transmit antenna beam still needs to be wide in order to illuminate the complete imaged swath. SCORE is especially attractive if implemented on-board, in which case it does not result in any substantial increase of the data rate, since the channels are combined on-board ${ }^{2}$. Viewed in terms of the tradespace, SCORE decouples the imaged swath width from the receiving antenna height.

SCORE is implemented by digitizing the signal induced on each element, multiplying by a time varying digital weight, and summing up the signals of all the channels. The weights can be understood as the beamforming coefficients which yield the required beam shape pointing in the changing direction-of-arrival of the echo while it traverses the ground. The resulting SCORE beam can be represented as:

$$
b[l, t]=\sum_{n=1}^{N_{e l}} s_{n}[l] \cdot w_{n}(t)
$$

where $s_{n}[l]=s_{n}\left(t_{l}\right)$ is the sampled signal induced on antenna feed element $n$, with $t_{l}=l / f_{s}$ the discrete sample time for a signal sampled at frequency $f_{s}$; and $w_{n}(t)$ is the complex weight function applied to the signal of channel $n$, where the time dependency on $t$ is to indicate that the weights change slower than the signal sampling $t_{l}$. The schematic hardware block diagram is shown in Fig. 1 (DBF unit 1).

Adopting matrix notation the sequence $s_{n}[l]$ is written as a column vector $\mathbf{s}_{n}=\left[s_{n}[0], \ldots, s_{n}\left[N_{r l}-1\right]\right]^{T}$, where $N_{r l}$ represent the total number of time samples of one range line and the signal matrix of all elevation channels becomes: $\mathbf{S}=\left[\mathbf{s}_{1}, \mathbf{s}_{2}, \ldots, \mathbf{s}_{N_{e l}}\right]$. Defining the $N_{e l}$ th element SCORE output beam vector, $\mathbf{b}(t)$ the vector equation corresponding to (1) becomes $\mathbf{b}(t)=\mathbf{S w}(t)$, where $\mathbf{w}(t)$ is the $N_{e l}$ th elements weight vector. Note that a strict matrix notation would require

\footnotetext{
${ }^{2}$ Indeed SCORE can be considered as a loss-less data compression along elevation [5].
}

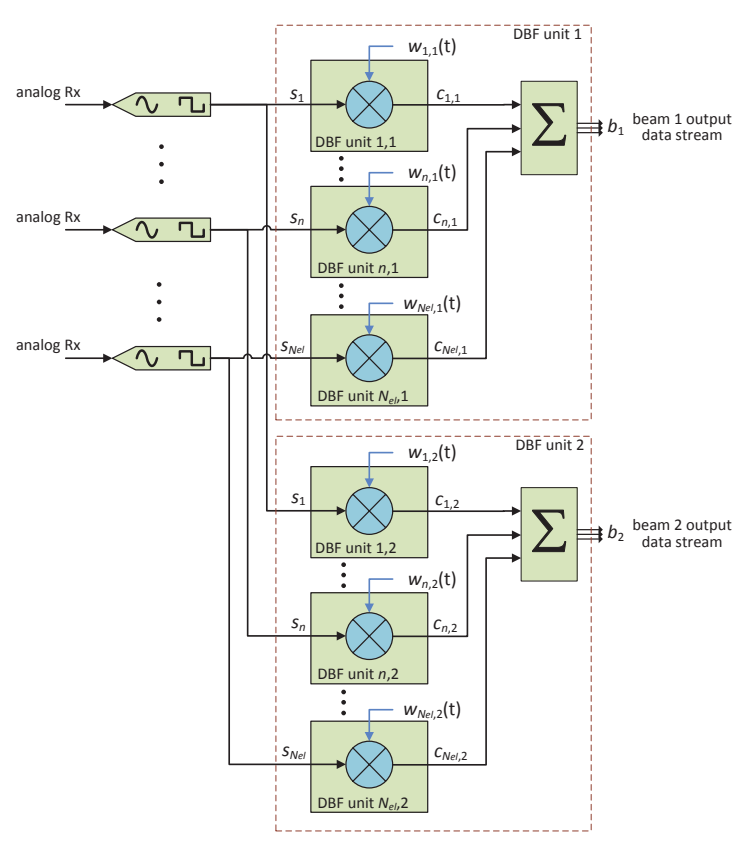

Fig. 1. Multi-Beam implementation for the example of $N_{\text {beam }}=2$ output beams. Here the signals of all feed elements are used to generate each output beam.

representing the weights through a block weight matrix, this is avoided here by writing the weight vector as a function of time.

The mathematical model applies to both planar and reflector antenna systems as the weights are applied to all $N_{e l}$ elevation channels. In the case of a reflectorantenna the set of feed array elements $\mathcal{N}_{\text {act }}$ on which the echo signal is induced are called active; a "clean" design would take this aspect into account by summing only the active channels' data streams.

\subsection{Multiple Azimuth Channels (MACs)}

By using multiple channels in along-track direction and a proper reconstruction processing [6] of the data, the azimuth resolution can be improved (in theory) to $\lambda / 2$. MACs basically increases the acquired Doppler bandwidth while ensuring sufficient azimuth sampling. The main feature, however, of MACs is, that it allows decoupling the azimuth resolution from the imaged swath width; overcoming one of the fundamental constraints of single channel SAR. As the system is acquiring data simultaneously with $N_{a z}$ azimuth channels, the hardware structure needs to be repeated $N_{a z}$ times. 


\section{MULTI-BEAM IMAGING}

A SAR instrument transmits a linearly frequency modulated pulse (chirp) of duration $\tau_{p}$ illuminating a wide swath on the ground. A pulse is transmitted every $T_{P R I_{0}}$ seconds (cf. Fig. 2 with $\Delta T_{i} \equiv 0$ ) where PRI is the Pulse Repetition Interval. For a swath of a few hundreds of kilometers the duration of the ground return $T_{\text {echo }}$ is larger than the time between the transmitted pulses $T_{P R I_{0}}$. As a consequence when imaging a wide swath multiple pulses are transmitted within the swath echo time $T_{\text {echo }}$ and thus multiple pulses traverse the swath simultaneously. In a conventional SAR these would be considered as unwanted range ambiguities, however here these are useful, i.e. wanted signals [7].

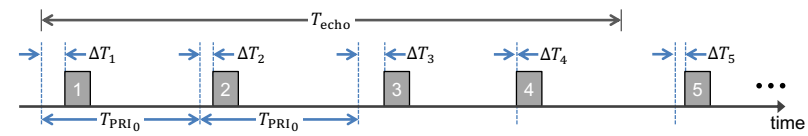

Fig. 2. Transmitted pulse sequence as a function of time. The duration of the ground echo return is $T_{\text {echo }}$.

In the multi-beam technique DBF is used to generate multiple simultaneous SCORE beams recording the echos of different pulses transmitted by a wide beam illumination, where the echos arrive from different directions. The received signal vector is thus multiplied by different sets of time varying weights corresponding to different output beams. Given the received signal vector $\mathbf{s}_{n}$ of feed element $n$ and defining the weight vector that generates the $k$ th output beam $\mathbf{b}_{k}(t)$ as $\mathbf{w}_{k}(t)=\left[w_{1, k}(t), w_{2, k}(t), \ldots, w_{N_{e l}, k}(t)\right]^{T}$ gives:

$$
\mathbf{b}_{k}(t)=\mathbf{S w}_{k}(t)=\sum_{n \in \mathcal{N}_{a c t, k}} \mathbf{s}_{n} w_{n, k}(t) .
$$

with $\mathcal{N}_{a c t, k}$ active elements of the $k$ th SCORE beam.

In Fig. 1 each feed element contributes to all $N_{\text {beam }}$ beams and thus the output of each DBF unit is the weighted sum of $N_{e l}$ feed elements. This structure is for a planar antenna system, in the case of a reflector system an "economical" design would connect each DBF unit only to a subset of the elements.

As the reception of the ground echo will be interrupted by the transmit events, blind ranges will occur. With the time-to-range mapping, namely $t=2 \mathrm{r} / \mathrm{c}$, these interruptions mask the reception of the echo of a specific range segment as illustrated schematically in
Fig. 3. For each transmit event a total of $\tau_{p} f_{s}$ samples in each azimuth channel will be lost, which is in the order of a few thousand samples. The ground extent for which $50 \%$ of the chirp bandwidth and power are lost is $\tau_{p} c / 2 \sin \eta_{i}$, where $\eta_{i}$ is the incidence angle.

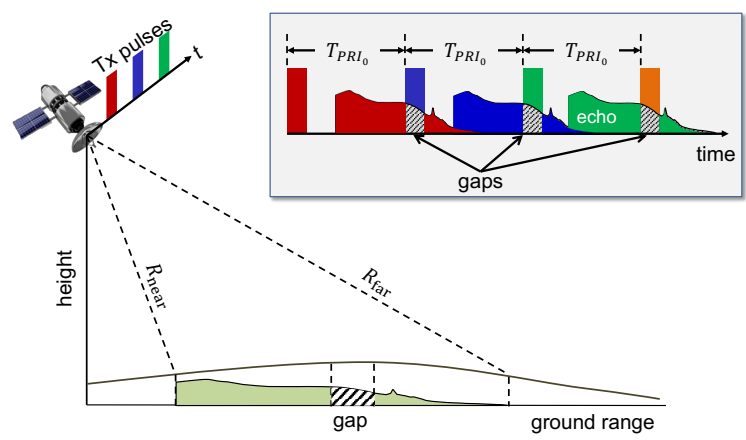

Fig. 3. Schematic representation of transmit events causing gaps in the received echos.

\section{STAGGERED SAR}

The SAR instrument successively transmits linearly frequency modulated pulses (chirps) of fixed duration $\tau_{p}$ at time instants $t_{i}=(i-1) \cdot T_{P R I_{0}}+\Delta T_{i}$ with the pulse index $i=1,2, \ldots$, and $\left|\Delta T_{i+1}\right|<T_{P R I_{0}}+\Delta T_{i}$ changes on a pulse-to-pulse basis according to a predefined sequence, which is repeated periodically every $N_{\text {seq }}$ pulses. This operation mode, known as Staggered SAR [8], where even though gaps still occur for every transmitted pulse, their position in the return echo will change for every transmitted pulse, as illustrated in Fig. 4. It is possible to design the delays $\Delta T_{i}$ of the sequence such that the gaps created by consecutive transmit pulses $i$ and $i+1$ do not overlap [8].

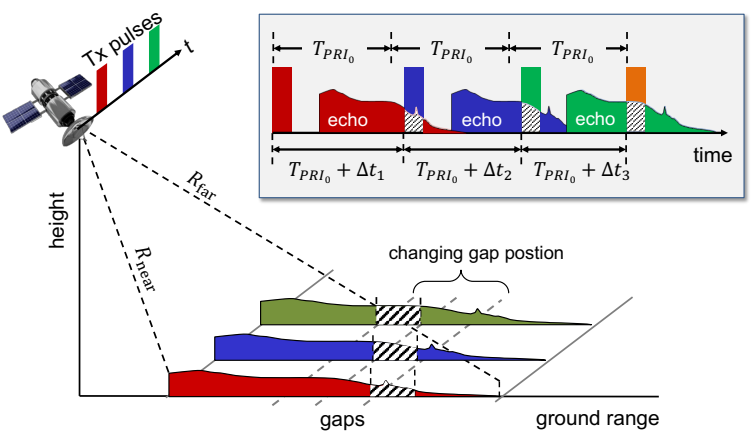

Fig. 4. Schematic representation of transmit events and received echos with PRI staggering. 
It is then possible to "remove" the gaps a posteriori by interpolating the values of the lost samples from the available signal support. This interpolation is in the azimuth direction as described in [8] using a Best Linear Unbiased Estimator (BLUE) for single channel SAR, and in [9] using a novel Virtual Beam Synthesis (VBS) approach for multiple azimuth channels. The staggered SAR operation has important implications for the sampling of the signal in azimuth: Obviously, as the pulse transmission instances are irregular, the respective spatial azimuth sampling is also non-uniform. This needs to be taken into account during SAR processing. The above mentioned methods $[8,9]$ take care both of the interpolation and azimuth regularization. A deeper information-theoretical understanding, c.f. [9], shows that azimuth samples from successive transmit pulses need to contain information which can be used to interpolate the missing gap samples; this requires a redundancy in the data, which translates into an azimuth oversampling.

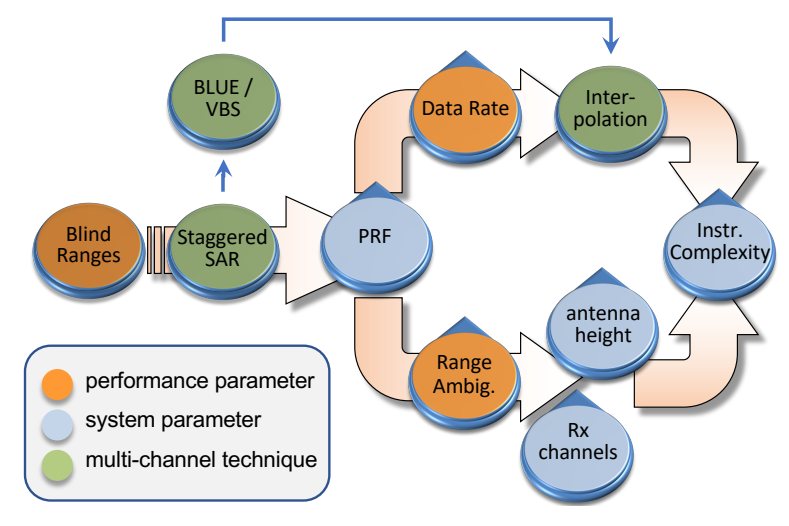

Fig. 5. The trade-space for staggered SAR operation.

A summary of the trade-space for staggered SAR operation is shown in Fig. 5. Starting point is a multibeam SCORE/MACs. To remove the gaps staggered SAR is introduced, causing additional azimuth processing complexity through the use of BLUE or VBS. Further, the necessary oversampling requires an increase in the Pulse Repetition Frequency (PRF) which is typically in the order of 1.5 to 2 ! This has a significant impact on the SAR performance, specifically in terms of range ambiguities and data rates. Mitigating the higher range ambiguities requires increasing the receive antenna height and additional elevation channels. The data rate can be reduced by suitable on-board interpolation processing, which in turn require the BLUE/VBS to be carried out on-board! This is a non-trivial task as the processing is along azimuth, thus operating on multiple echo data.

The net effect of staggered SAR is a non-negligible increase in the instrument complexity, antenna size, and on-board resources. As mentioned earlier, the alternative strategy is a multi-beam instrument and mission operations means to mitigate the gaps. Although more efficient, this does not come free of cost: the instrument operation requires a change of PRF on alternate observations and the time series of observations contains gaps.

\section{REFERENCES}

[1] M. Younis, P. López-Dekker, F. Bordoni, P. Laskowski, and G. Krieger, "Exploring the trade-space of MIMO SAR," in Proc. Int. Geoscience and Remote Sensing Symposium IGARSS'13, Melbourne, Australia, July 2013.

[2] G. Krieger, M. Zonno, J. Mittermayer, A. Moreira, S. Huber, and M. Rodriguez-Cassola, "MirrorSAR: A fractionated space transponder concept for the implementation of low-cost multistatic SAR missions," in Proc. European Conference on Synthetic Aperture Radar EUSAR'2018, Aachen, Germany, June 2018.

[3] M. Villano, M. Pinheiro, G. Krieger, A. Moreira, P. Rosen, S. Hensley, and C. Veeramachaneni, "Gapless imaging with the NASA-ISRO SAR (NISAR) mission: Challenges and opportunities of staggered SAR," in Proc. European Conference on Synthetic Aperture Radar EUSAR'2018, June 2018.

[4] M. Süß and W. Wiesbeck, "Side looking SAR system," U.S. Patent 6870 500, Mar., 2005.

[5] "Calibration and data reduction for digital beam forming instruments - concept discussion," European Space Agency (ESA/ESTEC), technical report D1, ESA ITT AO/1-8469/15/NL/FE, Mar. 2017.

[6] G. Krieger, N. Gebert, and A. Moreira, "Unambiguous SAR signal reconstruction from non-uniform displaced phase centre sampling," IEEE Geoscience and Remote Sensing Letters, vol. 1, no. 4, pp. 260-264, Oct. 2004.

[7] M. Younis, P. López-Dekker, and G. Krieger, "MIMO SAR operation modes and techniques," in Proc. European Conference on Synthetic Aperture Radar EUSAR'2014, Berlin, Germany, June 2014.

[8] M. Villano, G. Krieger, and A. Moreira, "Staggered SAR: High-resolution wide-swath imaging by continuous PRI variation," IEEE Transactions on Geoscience and Remote Sensing, vol. 52, no. 7, pp. 4462-4479, July 2014.

[9] F. Almeida, M. Younis, G. Krieger, and A. Moreira, "Multichannel staggered SAR azimuth processing," IEEE Transactions on Geoscience and Remote Sensing, vol. 56, no. 5, pp. 2772-2788, May 2018. 